\title{
A implantação da consulta de enfermagem ao paciente com insuficiência cardíaca
}

\section{The insertion of nursing assessment for a heart failure patient}

\section{La implantación de la consulta de enfermeria al paciente com insuficiencia cardiaca}

Ana Carla Dantas Cavalcanti', Dayse Mary da Silva Correia", Gisella de Carvalho Queluci"l

\author{
Profa Adjunta da Escola de Enfermagem Aurora de Afonso Costa da Universidade Federal Fluminense (EEAAC/UFF). Email: \\ ana_carladc@yahoo.com.br. \\ "Profa Assistente da EEAAC/UFF. Email: dayse.uff@oi.com.br. \\ II' Profa Assistente da EEAAC/UFF. Doutoranda em Enfermagem da Escola de Enfermagem Anna Nery/Universidade Federal do Rio de Janeiro. \\ Email: gisellaqueluci@yahoo.com.br.
}

\section{RESUMO}

Trata-se de relato de experiência do Projeto de Extensão "Consulta de Enfermagem ao Paciente com Insuficiência Cardíaca" (IC), desenvolvido com o apoio do Curso de Mestrado em Ciências Cardiovasculares e do Núcleo de Fundamentos de Enfermagem da Universidade Federal Fluminense(RJ). Tem como objetivo descrever sua implementação enquanto modalidade adequada e eficaz, para proporcionar ensino, pesquisa e assistência. O projeto é constituído das seguintes atividades: consulta de enfermagem baseada em NANDA-NIC-NOC(NNN); triagem, educação em saúde na sala de espera, desenvolvidas por docentes, enfermeiros e acadêmicos de enfermagem. O processo de cuidar permeia a identificação e compreensão das respostas dos pacientes com insuficiência cardíaca aos problemas de saúde reais e potenciais facilitando a escolha de intervenções de enfermagem. Assim, estimula medidas farmacológicas e não-farmacológicas que se propõem a melhorar a qualidade de vida e aderência ao tratamento através de educação em saúde.

Descritores: Enfermagem; Diagnóstico de enfermagem; Educação em saúde.

\section{ABSTRACT}

This article aimed at reporting the experience of the Project on Nursing advice for a Heart Failure Patient, developed with the support of the master course in "Cardiovascular Sciences" and the "Fundaments Nursing Nucleus" of the Federal Fluminense University (RJ). The objective is describing its insertion as an efficient and appropriate mean of teaching, research and assistance. The project consist of the following activities: nursing advice based on NANDA-NIC-NOC (NNN); a room where patient are guided to proper medical assistance; health education at the waiting room. These activities are accomplished by professors, nurses and students. The process of care alternates the identification and understanding of heart failure patient answers to real and potential health problems, making easy the choose of nursing interventions, promoting therefore pharmacological and no-pharmacological measures in order to guarantee to patient the quality of life and to increase his acceptance to the treatment through health education.

Descriptors: Nursing; Nursing diagnosis; Health education.

\section{RESUMEN}

Se trata de un relato de experiencias con respecto del Proyecto de Extensión “ Consulta de Enfermería al Paciente con Insuficiencia Cardiaca (IC), hecho con el apoyo del Curso Post Graduación en Ciencias Cardiovasculares y del Núcleo de Fundamentos de Enfermería de la Universidad Federal Fluminense (RJ). Tiene como objetivo describir su implantación como un medio adecuado y eficiente para proporcionar enseñanza, pesquisa y asistencia. El proyecto consiste de las siguientes actividades: consulta de enfermería basada en la NANDA-NIC-NOC; ala en que los pacientes son encaminados a consulta medica; y educación en salud en la sala de espera; actividades realizadas por profesores, enfermeros y estudiantes de enfermería. El proceso de cuidar alterna la identificación y comprensión de las respuestas de los pacientes con insuficiencia cardiaca relativas a los problemas de salud reales y potenciales, haciendo fácil la selección de intervenciones de enfermería. Así, estimulando medidas farmacológicas y no-farmacológicas para una mejor calidad de vida y mayor grado de adhesión al tratamiento por medio de la educación para la salud.

Descriptors: Enfermería; Diagnostico de enfermería; Educación en salud. 


\section{NTRODUÇÃO}

A Insuficiência Cardíaca se constitui em um dos principais problemas de saúde pública no mundo inteiro, apresentando uma prevalência de 1 a $2 \%$ da população, apesar do avanço clínico e tecnológico nesta área, nas últimas décadas. Em conseqüência, verifica-se um alto índice de internações, que ocasionam alto custo, bem como o aumento de reinternações, que têm sido atribuídas a não adesão ao tratamento farmacológico e às medidas nãofarmacológicas ${ }^{(1-2)}$.

Somente no Brasil, segundo dados do Sistema Único de Saúde (SUS) houve o registro de aproximadamente 398 mil internações por insuficiência cardíaca no ano de 2000 com 26 mil mortes, que correspondem a $30 \%$ de internações ${ }^{(1)}$.

Dessa forma, a meta de cuidado para o paciente com insuficiência cardíaca inclui estratégias de educação para subsidiar maior adesão ao tratamento e conseqüente bem estar e aumento da qualidade de vida, com diminuição de internações. Estas se fortalecem com atuação de equipe multidisciplinar, abordagem holística, foco nas necessidades humanas individuais e inclusão do familiar. Destaca-se, nesta equipe, a atuação do enfermeiro, que prioriza através de atendimento individualizado ou em grupo, a educação em saúde ${ }^{(3-7)}$.

Este artigo tem como objetivo descrever a experiência vivenciada no projeto de extensão "Consulta de Enfermagem ao Paciente com Insuficiência Cardíaca", enquanto modalidade adequada e eficaz, para proporcionar ensino, pesquisa e assistência, na unidade ambulatorial do Hospital Universitário Antônio Pedro(UFF/Niterói $\mathrm{RJ})^{(8)}$.

O projeto de extensão foi idealizado com o intuito de implementar a sistematização da assistência de enfermagem (SAE) a pacientes com insuficiência cardíaca em nível ambulatorial, buscando à documentação e organização dos dados coletados na consulta, além da intervenções pautadas em raciocínio clínico. De acordo com a resolução 272/2002 (COFEN), a SAE deve ser realizada em todas as instituições de saúde, sendo função privativa do enfermeiro. Esta é composta das seguintes fases: histórico de enfermagem, exame físico, diagnóstico de enfermagem, prescrição de enfermagem, evolução de enfermagem e relatório de enfermagem ${ }^{(9)}$.

A utilização da SAE desenvolve o pensamento crítico na prática de enfermagem e gera autonomia técnica, gerenciamento, individualização, uniformização, continuidade e avaliação do cuidado prestado através da promoção do cuidado humanizado, dirigido a resultados e de baixo custo, despertando nos enfermeiros o interesse contínuo de reavaliar suas atividades e decidir qual a melhor maneira de desempenhá-las ${ }^{(10)}$.
Para a elaboração dos diagnósticos de enfermagem é utilizada a Taxonomia de NANDA II (North American Nursing Diagnosis Association), que utiliza uma linguagem internacional para a classificação dos diagnósticos de enfermagem. A escolha por uma linguagem padronizada se deu pela associação do projeto com atividades de ensino e pesquisa. A NANDA possibilita o raciocínio clínico através do julgamento dos fatores relacionados e características definidoras. Logo, estimula o pensamento crítico dos acadêmicos de enfermagem e enfermeiros envolvidos no projeto as respostas dos pacientes com insuficiência cardíaca. Desta forma, permite direcionar as intervenções de enfermagem, que visam melhorar a qualidade de vida dos mesmos e diminuir os fatores que estão ocasionando as reinternações, bem como alcançar os resultados de enfermagem $^{\text {(11-12) }}$

Para a prescrição e implementação dos cuidados de enfermagem utilizamos a Classificação de Intervenções de Enfermagem (NIC), que é uma linguagem padronizada que visa o desenvolvimento de sistemas de informação, o ensino da tomada de decisões a acadêmicos, a determinação dos custos dos procedimentos oferecidos, o planejamento de recursos necessários, a utilização de uma linguagem para comunicar a função da enfermagem, além da articulação com os sistemas de classificação de outros provedores de cuidados de saúde ${ }^{(13)}$.

Para avaliar a qualidade dos cuidados de enfermagem implementados utilizamos a Classificação de Resultados de Enfermagem (NOC), que é uma linguagem comum de resultados específicos para a enfermagem que contribui de maneira objetiva na mensuração dos mesmos, ajudando na escolha das intervenções e fornecendo dados para evidências científicas, contribuindo para a eficiência da enfermagem e construção de inovações tecnológicas ${ }^{(14)}$.

Desde 2001 a NANDA tem negociado uma aliança com o Centro de Classificação da Faculdade de Enfermagem da Universidade de Iowa, EUA. Dessa aliança, explorou-se a possibilidade de desenvolver uma estrutura taxonômica comum, com os objetivos de tornar visível a relação entre as três classificações - diagnósticos, intervenções e resultados de enfermagem (NNN) - e de facilitar a ligação entre os três sistemas ${ }^{(13-15)}$. Para a consulta de enfermagem a pacientes com insuficiência cardíaca temos vivenciado a ligação entre as três classificações NANDA-NIC-NOC. Esta experiência tem nos possibilitado a exeqüibilidade de uma prática pautada em evidências científicas.

Observamos após a implantação da consulta de enfermagem, a necessidade de inserção das atividades de triagem e educação em sala de espera ao projeto de extensão. A triagem surgiu para uma avaliação clínica inicial e encaminhamento para o 
enfermeiro, ou outro profissional da equipe, sistematizando a entrada do paciente e organizando o direcionamento do mesmo. A educação em sala de espera visa utilizar o tempo no qual o paciente aguarda para as consultas para participação em estratégias variadas de ensino.

O processo de cuidar ao paciente com insuficiência cardíaca permeia a identificação e compreensão das respostas dos pacientes com insuficiência cardíaca aos problemas de saúde reais e potenciais facilitando a escolha de intervenções de enfermagem. Estimula, assim, medidas farmacológicas e não-farmacológicas que se propõem a melhorar a qualidade de vida e aderência ao tratamento através de educação em saúde.

Trata-se de um relato de experiência da implantação do Projeto de Extensão "Consulta de Enfermagem ao Paciente com Insuficiência Cardíaca", desenvolvido pelos docentes da Escola de Enfermagem Aurora de Afonso Costa (EEAAC/UFF), acadêmicos de enfermagem e enfermeiros voluntários.

\section{DESCREVENDO O CENÁRIO E A PROPOSTA}

Em agosto de 2006 foi iniciado o processo de implantação da consulta de enfermagem para o paciente com insuficiência cardíaca no ambulatório do Hospital Universitário Antônio Pedro (HUAP), em parceria com o Programa de Pós-Graduação em Ciências Cardiovasculares da Faculdade de Medicina e com o Núcleo de Fundamentos de Enfermagem da Escola de Enfermagem Aurora de Afonso Costa, ambos da Universidade Federal Fluminense.

O Programa de Pós-Graduação em Ciências Cardiovasculares já realizava atendimento médico a pacientes com insuficiência cardíaca no ambulatório. Até então, a equipe de enfermagem atuava no na administração do espaço físico.

A implantação da consulta de enfermagem teve como objetivo geral implementar cuidados específicos de enfermagem a pacientes do ambulatório de insuficiência cardíaca do HUAP, orientando-os no tratamento não-farmacológico e farmacológico, na tentativa de estimular o autocuidado, aumentar a adesão ao tratamento, melhorar a qualidade de vida e diminuir o índice de hospitalização.

Foram definidos os seguintes objetivos específicos: elaborar um instrumento para a realização da consulta de enfermagem de acordo com a Resolução 272/2002 do COFEN, baseado nas classificações NANDA-NIC-NOC; implementar a consulta de enfermagem para os pacientes com insuficiência cardíaca em acompanhamento ambulatorial no HUAP; desenvolver atividades de educação em saúde em sala de espera; realizar a triagem para as consultas de enfermagem, médica, nutricional e de fisioterapia; contribuir para a aprendizagem de acadêmicos de enfermagem sobre
SAE, classificações NNN e IC, ampliar o conhecimento teórico-prático de enfermeiros e acadêmicos de enfermagem.

\section{A consulta de enfermagem}

Para a implantação da consulta de enfermagem primeiramente foi elaborado um formulário baseado nas classificações da NANDA-NIC-NOC. Este tem por finalidade documentar e comunicar aos outros membros da equipe de saúde a evolução e a condição do paciente. O formulário inclui o histórico de enfermagem direcionado aos domínios, classes e diagnósticos de enfermagem propostos pela Taxonomia II da NANDA, como forma de reconhecer um sistema de documentação compreensível e dinâmico, registrando mais dados em menos tempo e espaço.

O histórico de enfermagem compreende a fase do processo de enfermagem, na qual os enfermeiros coletam os dados subjetivos e objetivos do paciente. Ele enfoca a determinação do estado atual de saúde do paciente e sua capacidade de funcionamento. Para a investigação de enfermagem ao paciente com insuficiência cardíaca, os enfermeiros e acadêmicos de enfermagem obtém dados entrevistando, observando, examinando, analisando o prontuário com o registro das informações dos médicos, nutricionistas e fisioterapeutas e resultados de exames, como ecocardiograma, ECG, bioquímica, entre outros ${ }^{(10)}$.

É importante ressaltar que o histórico de enfermagem foi baseado nos domínios da NANDA, compreendendo a busca de informações subdivididas como a seguir: promoção da saúde, nutrição, eliminação e troca, atividade e repouso, percepção e cognição, auto-percepção, relacionamento de papel, sexualidade, enfrentamento/tolerância ao estresse, princípios de vida, segurança/proteção, conforto e crescimento/desenvolvimento. A opção por um roteiro para o histórico classificado em domínios visa fornecer um parâmetro inicial para acadêmicos de enfermagem ${ }^{(11)}$.

Após a entrevista e o exame físico, são elaborados os diagnósticos de enfermagem e selecionadas as intervenções e resultados direcionados as necessidades individuais de cada paciente. Alguns questionamentos são orientados aos acadêmicos para a tomada de decisão clínica e julgamento das respostas deste paciente, como(11${ }^{13)}$ : O paciente tem algum problema que exige intervenção de enfermagem? O paciente tem conhecimento da doença e seu tratamento? De que forma a insuficiência cardíaca tem afetado sua vida diária? Que resultados pretendo alcançar para este paciente?

As consultas de enfermagem são realizadas semanalmente às segundas-feiras, das 15:00 às 19:00, por enfermeiros e acadêmicos de enfermagem 
sob a supervisão das docentes. Durante a mesma, os profissionais identificam diagnósticos de enfermagem, selecionam as intervenções de enfermagem e avaliam os resultados.

A primeira consulta é mais longa e tem duração de aproximadamente uma hora. As subseqüentes duram em torno de 30 minutos. Durante a consulta de enfermagem a fala do paciente é respeitada e o enfermeiro ouve atentamente para que ele possa expressar suas angústias e tirar as dúvidas sobre a doença e tratamento farmacológico e nãofarmacológico. O ambiente é silencioso e preserva a privacidade do mesmo.

\section{Educação em saúde na sala de espera}

A sala de espera visa à educação em saúde aos pacientes que estão aguardando a consulta da equipe multidisciplinar (enfermeiros, médicos, nutricionista e fisioterapeuta). A equipe de enfermagem trabalha na orientação de temas relacionados ao tratamento da insuficiência cardíaca com o objetivo de ensinar os pacientes sobre a doença, autocuidado, tratamento e qualidade de vida. Os temas principais da sala de espera são: insuficiência cardíaca, manejo da doença (autocuidado), tabagismo, alcoolismo, nutrição, atividade física, sono e repouso, tratamento farmacológico e não-farmacológico.

O ensino da doença e seu tratamento tem sido amplamente discutido como intervenção de enfermagem $^{(13)}$. Assim, os temas são abordados com linguagem simples para facilitar o entendimento dos pacientes com estratégias diferenciadas, como, palestras, jogos e dramatizações.

Os pacientes respondem a um pré-teste e pósteste do tema, ou seja, um questionário com perguntas, a serem respondidas antes e após as atividades, para avaliar o entendimento sobre o conteúdo e orientações de enfermagem.

\section{Triagem}

A criação do serviço de triagem foi imprescindível, uma vez que várias dificuldades foram identificadas no momento de seleção dos pacientes para a consulta de enfermagem, tais como: a não disponibilidade de tempo (relatada pelos pacientes) para a consulta de enfermagem; a falta de conhecimento dos mesmos sobre a importância da consulta com os enfermeiros; comunicação inadequada entre os profissionais da equipe multidisciplinar acerca do encaminhamento dos pacientes para a consulta de enfermagem; dificuldades dos enfermeiros sobre a respectiva ordem das consultas dos demais profissionais de saúde.

Desta forma, a triagem foi organizada com os seguintes objetivos: organizar o atendimento dos pacientes no ambulatório; encaminhar os pacientes para a consulta médica, de enfermagem, nutrição e fisioterapia; identificar as queixas principais, verificar sinais vitais, peso, altura, Índice de Massa Corporal (IMC), entre outras observações; realizar o controle de atendimento dos pacientes e agendamento das consultas de enfermagem; elaborar fichas de atendimento e controle de marcação de consultas da equipe multidisciplinar.

O fluxograma, a seguir, ilustra o serviço de triagem e as demais consultas onde o paciente é encaminhado.

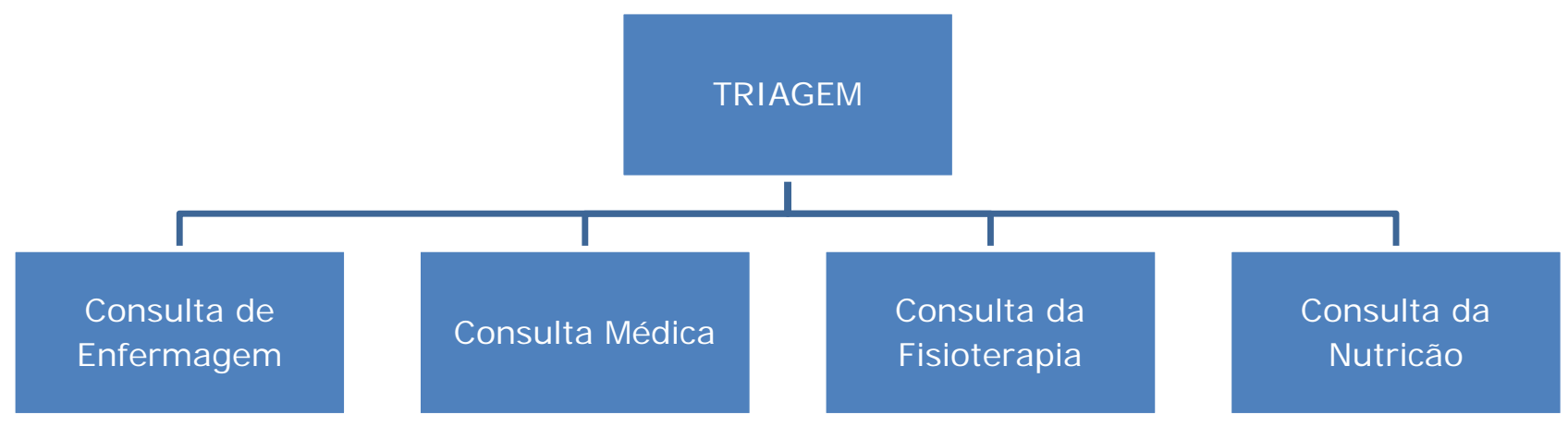

Figura 1: Serviço de atendimento na triagem; Niterói, 2007.

Ressalta-se que os pacientes são atendidos primeiramente na triagem e, posteriormente, encaminhados aos demais profissionais, de acordo com o controle de marcação de consultas.

Além das atividades direcionadas aos pacientes, foi criado em outubro de 2006, o Grupo de Estudos de Sistematização da Assistência de Enfermagem e
Insuficiência Cardíaca (GESAEIC), o qual se reúne mensalmente para discussão de artigos científicos, avaliação, planejamento mensal de atividades a médio e longo-prazo e troca de informações científicas dos seguintes temas: Sistematização da Assistência de Enfermagem, Classificações, 
Tratamento farmacológico e não-farmacológico da insuficiência cardíaca e exame físico.

A dinâmica atribuída ao grupo perpassa por um endereço eletrônico, onde são trocadas informações sobre artigos, agenda de reuniões, confirmação de atividades semanais, quinzenais e mensais.

Os membros do GESAEIC também participam do Clube de Insuficiência Cardíaca, quando têm oportunidade de debater situações clínicas e artigos científicos com profissionais e acadêmicos de enfermagem, medicina, fisioterapia, nutrição e biologia.

O GESAEI C realiza um seminário por ano para a divulgação e discussão das temáticas com enfermeiros, docentes e pesquisadores sobre SAE e IC, quando temos a oportunidade de estabelecer contato com profissionais da equipe multidisciplinar. Nos anos de 2007 e 2008 tivemos convidados das seguintes instituições: INCOR-SP, UERJ, INCL e UFRJ .

\section{Avaliação}

A extensão universitária tem sido vivenciada de maneira rica para o ensino, assistência e pesquisa, por apresentar uma experiência inovadora de integração, que agrega graduandos com pósgraduandos de enfermagem, medicina, fisioterapia e nutrição, todos com uma meta em comum, que é a prestação de assistência ao paciente com insuficiência cardíaca.

No ensino temos tido a oportunidade de receber acadêmicos de enfermagem como voluntários para colaborar na execução das atividades de triagem, educação em sala de espera e consulta de enfermagem. Estes têm participado das discussões científicas e aprimorado o conhecimento na temática.

Através do contato com os pacientes com insuficiência cardíaca os acadêmicos são estimulados a resolução de problemas através da investigação científica. Assim, estes têm realizado seus trabalhos de conclusão de curso, trabalhos de monitoria e outras pesquisas com problemáticas vivenciadas no projeto. Os estudos realizados têm sido apresentados em eventos científicos, como Congresso da Sociedade de Cardiologia do Estado do rio de Janeiro, Pesquisando em Enfermagem, Encontro UFF/INCOR, Congresso do Grupo de Estudo de Insuficiência Cardíaca, Congresso Brasileiro de Enfermagem, Simpósio Nacional de Diagnóstico de Enfermagem, Semana Científica da EEAAC/UFF, Semana de Enfermagem da UFF, Semana de Extensão da UFF, Fórum Internacional de Cardiologia em Enfermagem, entre outros.

Mais de 200 pacientes já foram beneficiados com as atividades desenvolvidas na triagem, educação em sala de espera e consultas de enfermagem. Os mesmos recebem orientações em grupo e individuais. Os familiares também participam das atividades desenvolvidas, sendo também beneficiados pelas orientações recebidas. Todos os pacientes recebem um folder educativo com ilustrações sobre insuficiência cardíaca.

Como limitações para a implantação e execução do projeto de extensão temos a escassez de recursos materiais, que tem dificultado a confecção de materiais, a informatização do banco de dados e a elaboração de materiais para as atividades de educação em sala de espera.

\section{CONSI DERAÇÕES FI NAIS}

A Insuficiência Cardíaca é hoje uma condição endêmica, sendo a primeira causa de internação hospitalar em idosos no Brasil e em Niterói, apresentando elevada morbimortalidade. A implementação da consulta de enfermagem tem proporcionado a identificação e compreensão das respostas dos pacientes com insuficiência cardíaca aos problemas de saúde reais e potenciais, facilitando a escolha de intervenções que são uma alternativa de estimular medidas não-farmacológicas para melhorar a qualidade de vida através de educação para saúde, com orientações sobre alimentação, atividade física, posições de conforto, entre outras questões que possam auxiliá-los a manter uma vida mais saudável e aumentar a aderência ao tratamento.

O Projeto de Extensão Consulta de Enfermagem ao Paciente com Insuficiência Cardíaca amplia a perspectiva clínica para uma visão holística, que visa maior adesão ao tratamento e diminuição da internação hospitalar. No entanto, temos percebido que a proposta tem nos dado a oportunidade de ampliar o conhecimento científico através de pesquisas que possam estar utilizando o banco de dados, concretizado através da SAE.

Dessa forma, a consulta de enfermagem sistematizada traz benefício à comunidade, pois oferece atendimento de qualidade, que respeita a individualidade de cada paciente, na identificação de diagnósticos e na escolha das intervenções e avaliação dos resultados das mesmas,além de obter dados que ampliam a pesquisa em enfermagem na linha da SAE e IC, solidificando a possibilidade de basear as intervenções de enfermagem a essa clientela em evidências científicas.

Por meio das discussões em grupos e interação de enfermeiros, médicos, fisioterapeutas, nutricionistas, docentes e acadêmicos das respectivas áreas, se está produzindo o conhecimento de forma multidisciplinar, o que tem proporcionado a todos os membros do grupo uma experiência diversificada, única e solidária do saber e fazer em saúde.

\section{REFERÊNCI AS}

1. DATASUS [Internet]. Brasília: Ministério da Saúde (BR) [cited 2009 mar 10]. Departamento de Informática do SUS - DATASUS. Available from: http://w3. datasus.gov.br/datasus/datasus.php. 
2. Rabelo ER, Aliti GB, Domingues FB, Ruschel KB, Brun AO. O que ensinar aos pacientes com insuficiência cardíaca e porquê: o papel dos enfermeiros em clínicas de insuficiência cardíaca. Rev Latino-am Enfermagem [Internet]. 2007 [cited 2009 feb 16];15(1):165-70. Available from: URL: http://www.scielo.br/pdf/rlae/v15n1/pt_v15n1a24.pdf.

3. Barreto ACP, Drumond Neto C, Mady C, Alburquerque DC, Brindeiro Filho DF, Braile DM. Revisão das II Diretrizes da Sociedade Brasileira de Cardiologia para o Diagnóstico e Tratamento da Insuficiência Cardíaca. Arq. Bras. Cardiol. [Internet]. 2002;79 Suppl 4:1-30. Available from: http://www. scielo.br/pdf/abc/v79s4/a01v79s4.pdf.

4. Timmins F. Cardiac nurses' views of continuing professional education. Eur J Cardiovasc Nurs [Internet]. 2008 [cited 2009 feb 16];7(1):59-66. Available from: http://download.journals. elsevierhealth.com/pdfs/jou rnals/1474-5151/PIIS1474515107001764.pdf.

5. Dal EC, Bondiani AE, Zanolla L, Vassanelli C. Nurse educational activity on non-prescription therapies in patients with chronic heart failure. Eur J Cardiovasc Nurs [Internet]. 2007 [cited 2009 feb 16];6(4):314320. Available from: http://www. escardiocontent.org/periodicals/ejcn/artic le/S1474-5151(07)00143-0/abstract.

6. Lupón J, Gonzáles B, Mas D, Urrutia A, Arenas M, Domingo $M$ et al. Patients self-care improvement with nurse education intervention in Spain assessed by the European heart failure self-care behaviour scale. Eur J Cardiovasc Nurs [Internet]. 2008 [cited 2009 feb 16];7(1):16-20. Available from: http://download.journals. elsevierhealth.com/pdfs/jou rnals/1474-5151/PIIS1474515107001661.pdf.

7. Falk S, Wahn AK, Lidell E. Keeping the maintenance of daily life in spite of chronic heart failure. A qualitative study. Eur J Cardiovasc Nurs [Internet]. 2007 [cited 2009 feb 16];6(3):192-199. Available

from:

http://www. escardiocontent. org/periodicals/ejcn/artic le/S1474-5151(06)00159-9/abstract.

8. Lima AFC, Kurcgant P. O processo de implementação do diagnóstico de enfermagem no Hospital Universitário da Universidade de São Paulo. Rev. esc. enferm. USP. 2006; 40(1):111-6.

9. Conselho Federal de Enfermagem. Resolução COFEN-272 de 27 de agosto de 2002. Dispõe sobre a Sistematização da Assistência de Enfermagem -SAEnas Instituições de Saúde Brasileiras. Rio de Janeiro (Brasil): COFEN; 2002.

10. Alfaro Lefevre, R. Aplicação do Processo de Enfermagem: promoção do cuidado colaborativo. 5th ed. Porto Alegre: Artmed; 2005.

11. North American Nursing Diagnosis Association (NANDA). Diagnósticos de Enfermagem da NANDA: Definições e Classificação. 2005-2006. Porto Alegre: Artmed; 2006.
12. International Council of Nurses. The International Classification for Nursing Practice $\left(\right.$ ICNP $\left.^{\circledR}\right)$ : a unifying framework. The Alpha Version. Geneva (Switzerland): International Council of Nurses; 1996. 13. Closkey JC, Bulechek GM. Classificação das Intervenções de Enfermagem (NIC). 3rd ed. Porto Alegre: Artmed; 2004.

14. Johnson M, Maas M, Moorhead S. Classificação dos Resultados de Enfermagem (NOC). 2nd ed. Porto Alegre: Artmed; 2004.

15. Johnson $M$ et al. Diagnósticos, resultados $e$ intervenções de enfermagem: ligações entre NANDA, NOC e NIC. Porto Alegre: Artmed; 2005.

Artigo recebido em 25.11.07.

Aprovado para publicação em 31.03.09. 